\title{
High Performance MEMS Sensor for Low Dew Point Humidity Detection
}

\author{
Anis Zribi ${ }^{1}$, Aaron Knobloch ${ }^{1}$, Wei-Cheng Tian ${ }^{1}$, Gerald Schultz \\ ${ }^{1}$ Micro and Nano Structures Technologies \\ General Electric Global Research Center, \\ ${ }^{2}$ General Eastern Instruments \\ Wilmington, MA 01887
}

Niskayuna, NY 12309

\begin{abstract}
A highly sensitive micro-machined humidity-sensor using novel detection techniques have demonstrated the ability to measure humidity within the range of $6 \mathrm{ppmw}\left(0.04 \% \mathrm{RH},-60^{\circ} \mathrm{C}\right.$ $\mathrm{DP})$ to $11 \mathrm{pptw}\left(60.7 \% \mathrm{RH}, 15^{\circ} \mathrm{C} \mathrm{DP}\right)$ at ambient with a resolution of approximately $2 \mathrm{ppm}$, a sensitivity of $69.6 \mathrm{~Hz} /{ }^{\circ} \mathrm{C} \mathrm{DP}$, a non linearity of $0.14 \%$ and a low hyteresis of approximately $0.07 \%$. This sensor operates in two distinct schemes where the measurand is either the heat or mass exchange associated with the adsorption/desorption of water vapor onto a hygroscopic polystyrene sulfonic acid thin film. While operation in both modes has been demonstrated, this paper will focus on the resonant sensor concept and performance. The sensor consists of two dual freestanding rectangular silicon nitride membranes with a $\mathrm{Pt} / \mathrm{Ti}$ current carrying conductor, stretched on top of a silicon frame. Only one of the two membranes is loaded with the sensing film. The second membrane is designed for use as a reference cell to compensate for noise parameter effects (e.g. pressure, temperature...etc). The membrane is driven into resonance using out-of-plane Lorentz forces, which are generated due to interaction of an external magnetic field with the AC powered conductor. Because of its low power consumption (less than $140 \mu \mathrm{W}$ ) and its high performance over a broad range of humidity, the sensor appears to be suitable for many applications ranging from industrial gas monitoring to medical diagnosis where there is a growing need for miniaturized gas and vapor sensors with lower detection limits while maintaining short response times and high chemical stability.
\end{abstract}

\section{INTRODUCTION}

The increasing need and the more stringent requirements for humidity sensors in applications such as process control, appliances, microelectronics, semiconductor, medical applications, and automotive have been continuously driving research and development of novel and high performance devices and sensor materials [1]. The areas of improvement for humidity sensors typically include sensor material stability, fouling, hysteresis, response speed, humidity range, operating environment, power consumption, size, and cost. Traditionally, these performance issues have been addressed by a wealth of sensing materials and transducers designed for specific applications with various properties. Accordingly, even the best of these sensors suffer from limitations regarding the sensitivity ranges, chemical stability, response speed, size, or simplicity.

A recent review by Rittersma [2] discussed the five main transduction techniques of state-of-the-art humidity sensors: capacitive, resistive, hygrometric, gravimetric, and optical. Among these transduction mechanisms, capacitive, gravimetric, and optical methods appear to be the best suited for low dew point humidity sensing considering their high sensitivity, low response times, low power consumption, and miniaturizability. Other sensing methods have shown promise in detection of humidity at low dew points [3]. MEMS-based batch fabrication techniques can significantly reduce sensor cost and allow for integrated electronics.

The majority of commonly used transducers for humidity sensing are based on capacitive concepts. These approaches are based on the measurement of the change in dielectric properties and/or dimensions of hygroscopic thin films upon absorption of moisture. Both resonant [4] and non-resonant [5] sensors have been built using this concept. However, regardless of the resonant nature of these sensors, their performance is primarily determined by the material properties and the electrode geometry.

Porous ceramics [6], high-humidity-uptake dielectric polymers and polymer composites [7] are the most frequent sensor materials used in capacitive humidity sensors. More recently, porous silicon [2] has been introduced for specific applications. While porous ceramics and silicon appear very attractive because of their large surface area, long-term structural stability, and their batch manufacturability, they suffer serious limitations in comparison to polymers in terms of response linearity, drift due to chemisorption of hydroxyl groups, and susceptibility to contaminants such as smoke.

Although capacitive transducers, in general, have demonstrated fast responses and high sensitivities on the order of a few hundred percent as published in the literature [1], the response time and signal to noise ratios of capacitive humidity sensors are still the main areas where improvements are required. According to Nitta and Cunningham $[6,8]$, the response time of capacitive sensors is dictated by the kinetics of water absorption, which is a diffusion-limited mechanism initiated by capillary condensation. The indication is that minimizing the response time of a sensor requires optimization of the material and transducer response simultaneously.

Numerous gravimetric humidity sensors are described in the literature with various operation mechanisms and performances [2, 9]. These devices measure the effect of a combination of sensor material, mass change and/or mechanical properties variation, induced by humidity uptake. The change in the sensor material properties produces a change in the resonance frequency of the sensor, which is the direct output of the sensor. Quartz Crystal Microbalances (QCMs), capacitive and piezoelectric Flexural Wave Plates (FPWs), capacitive nano/micro cantilevers and magnetoelastic films and ribbons are the most widely known transducers used in gravimetric humidity sensors. QCMs and capacitive plates/cantilevers are excited and driven into resonance by electrical energy whereas magnetoelastic sensors take magnetic energy as an input. Because of well-understood $[\mathbf{2}, \mathbf{1 0}]$ technical considerations, the bandwidth of these sensors is fairly narrow. QCMs, for example, operate at $\mathrm{MHz}$ frequencies with a total resonance peak shift for the entire $\mathrm{RH}$ range on the order of a $\mathrm{kHz}$ or less. As a result, these devices rely on complex and specific electronics to achieve high sensitivities and high signal to noise ratios. In contrast, the device presented in this paper is a gravimetric sensor designed to achieve high bandwidth, high sensitivity at a low cost. 


\section{THEORY}

The performance of resonant, gravimetric humidity sensors depends equally on the performance of the resonant and the chemical transducers. In an effort to develop a complete transfer function relating the performance of our sensor to all important variables (i.e. geometry, materials properties...etc), we created an analytical and a numerical model of the membrane and current carrying conductor system shown in Figure 1. The membrane is a low stress silicon nitride membrane, which is uniformly stretched by a tension $\mathrm{T}$ (force per unit length) from all four sides. The tension $\mathrm{T}$ is the result of the residual stress $\sigma$ built into the membrane during processing as described in the experimental section. The membrane has a mass $m$, a density $\rho$, an elastic modulus $E$, a Poisson ratio $v$ and it has the dimensions $a, b$, and $h$ as displayed in Figure 1. The boundary of the membrane, where the silicon frame is attached to the nitride, is treated as a Dirichlet boundary with zero displacement along the edges.
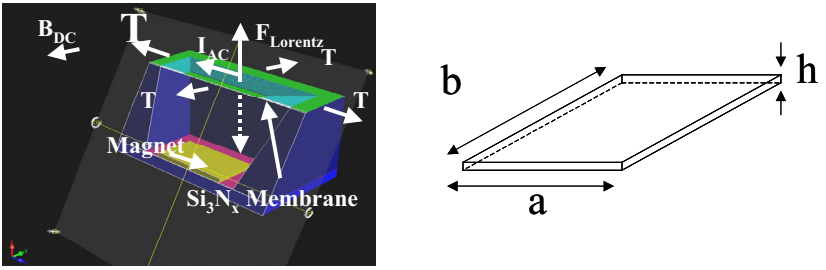

Figure 1. Operating principles of the sensor and its geometry.

In the absence of external forces, the wave equation describing the motion of the membrane is

$$
\mu \frac{\partial^{2} \delta}{\partial t^{2}}=-D \nabla^{4} \delta+T \nabla^{2} \delta, \quad \delta=\delta(x, y, t)
$$

where $\delta$ is the out of plane displacement of point $M$ on the membrane identified by it coordinates $x$ and $y$ in the selected cartesian reference. The mass per unit area, $\mu$, of the membrane is defined as

$$
\mu=\frac{m}{a b}=\rho h
$$

and $D$ describes the flexural rigidity of the membrane as defined by

$$
D=\frac{E h^{3}}{12\left(1-v^{2}\right)} .
$$

The boundary conditions of the membrane are zero displacement Dirichlet conditions and are expressed by the following

$$
\begin{aligned}
& \delta(0, y, t)=0, y \leq b \\
& \delta(a, y, t)=0, y \leq b \\
& \delta(x, 0, t)=0, x \leq a \\
& \delta(x, b, t)=0, x \leq a
\end{aligned},
$$

and the initial condition is a uniformly distributed mechanical perturbation

$$
\delta(x, y, 0)=\delta_{0}, 0 \leq y \leq b \text { and } 0 \leq x \leq a
$$

Using the method of separation of variables, we can express $\delta$ by

$$
\delta(x, y, t)=f(X) g(Y) \tau(t),
$$

where $f, g$, and $\tau$ are sinusoidal functions. By plugging this expression back into equations 1,4 , and 5 , we can solve for the resonance frequency of the first fundamental mode of the membrane:

$$
f_{r}=\sqrt{\frac{\pi^{2} E h^{2}}{48 \rho\left(1-v^{2}\right)}\left(\frac{1}{a^{2}}+\frac{1}{b^{2}}\right)^{2}+\frac{T}{4 \rho h}\left(\frac{1}{a^{2}}+\frac{1}{b^{2}}\right)} .
$$

According to (Eq.7), the resonance frequency of the membrane is a function of the residual stress in the membrane as well as the mass of the membrane. The cumulative effect of mass and stress change of the membrane is the basis of our sensing scheme. In fact, the polystyrene sulfonate $\left(\mathrm{H}^{+}\right.$state), a hygroscopic film, is a polyelectrolyte that swells considerably upon uptake of moisture in addition to a mass increase on order of $20 \% \mathrm{wt}$ at equilibrium. The swelling mechanism and the high humidity uptake of the sensor film have been discussed extensively in the literature [11].

The sensitivity of the transducer to mass $\left(S_{m}\right)$ and stress change $\left(S_{s t}\right)$ can be derived from (Eq.7). The differential of the resonance frequency with respect to mass and tension is related to these sensitivities by

$$
\begin{aligned}
d f_{r}= & \left(\frac{1}{2 m}\right)\left(-f_{r} d m+\frac{a b}{4}\left(\frac{1}{a^{2}}+\frac{1}{b^{2}}\right) \frac{1}{f_{r}} d T\right) . \\
& =S_{m} d m+S_{T} d T
\end{aligned}
$$

The cross sensitivity of this transducer to mass and stress variations is then expressed by

$$
\begin{aligned}
S_{\text {transducer }} & =\left|S_{m} * S_{T}\right| \\
& =\frac{1}{16 \rho^{2}{ }_{S i_{3} N_{x}}} \frac{1}{a b h^{2}}\left[\frac{1}{a^{2}}+\frac{1}{b^{2}}\right] .
\end{aligned}
$$

The indication of this simple model is that thinner membranes and smaller membranes have a higher sensitivity than larger and thicker diaphragms.

\section{DEVICE DESIGN \& FABRICATION}

The micromachined humidity sensor consists of two parts: silicon micromachined resonant membrane and the sensing material. The platform contains dual rectangular silicon nitride membranes with the metal conductor on top of it. The sensing material is applied to the backside of the membrane in the sensing cell but no sensing film is required for the reference cell. The reference cell is designed to compensate for the cross-sentivity to noise parameters (e.g. pressure, temperature, etc). Different sensing materials, such as polystyrene sulfonic acid (PSSA) or a

Table 1. Design of experiment examining the role of device geometry.

\begin{tabular}{|c|c|c|c|c|}
\hline Design & $\begin{array}{c}\text { Metal } \\
\text { width } \\
(\mu \mathrm{m})\end{array}$ & $\begin{array}{c}\text { Metal } \\
\text { length } \\
(\mu \mathrm{m})\end{array}$ & $\begin{array}{c}\text { Final Membrane Size } \\
(\mu \mathrm{m})\end{array}$ & $\begin{array}{c}\text { Chip Size } \\
(\mu \mathrm{m})\end{array}$ \\
\hline $1 / 2 \times$ Straight & 150 & 2005 & $1500 \times 1800$ & $6650 \times 6650$ \\
\hline $1 / 2$ X Serpentine & $50 / 50$ & 21505 & $1500 \times 1800$ & $6650 \times 6650$ \\
\hline $1 / 3$ X Straight & 100 & 2003 & $1000 \times 1300$ & $6650 \times 6650$ \\
\hline $1 / 3$ X Serpentine & $35 / 35$ & 15653 & $1000 \times 1300$ & $6650 \times 6650$ \\
\hline
\end{tabular}


functionalized self-assembled monolayer, have been used to maximize the device sensitivity.

This study examines the effects of the membrane dimension on sensor performance. The geometrical design of experiment has been performed to optimize the performance of the micromachined humidity sensors, as shown in Table 1 . The straight or serpentine microresistor design can be used for either resonance frequency shift or differential scanning nanocalorimetry measurement. For the resonance frequency shift experiment, the straight-line design is preferred because it can generate a larger Lorentz force and stable vibration modes. The metal dimension determines the electrical current passing through the conductor and thus, modulates the Lorentz force at a given magnetic flux. The dimensions of the membrane control the coverage area for the sensing material. The two contact pads are used to flow current through the conductor.

A relatively simple and robust process has been developed based on bulk micromachining. A layer of low stress silicon nitride $(\sim 200 \mathrm{MPa})$ with a thickness of 0.5 or $1 \mu \mathrm{m}$ is deposited on the surface of the $300 \mu \mathrm{m}$ thick (100) n-type silicon substrate. The current carrying conductor and contact pad consist of $5 / 40 \mathrm{~nm}$ thick $\mathrm{Ti} / \mathrm{Pt}$, which are patterned via a metallization and lift-off process. Finally, the etch windows are opened at the backside of the wafer using Reactive Ion Etching of silicon nitride and a 30\% Potassium Hydroxide $(\mathrm{KOH})$ etch with a rate of $1.1 \mu \mathrm{m} / \mathrm{min}$ at $80^{\circ} \mathrm{C}$, to release the silicon nitride membranes. During the $\mathrm{KOH}$ etching, the front side of the wafers is protected by a special wafer holder so the $\mathrm{KOH}$ will not attack the metal conductor. This process has a yield of approximately $100 \%$. The inset of Figure 2 shows a final packaged humidity sensor.

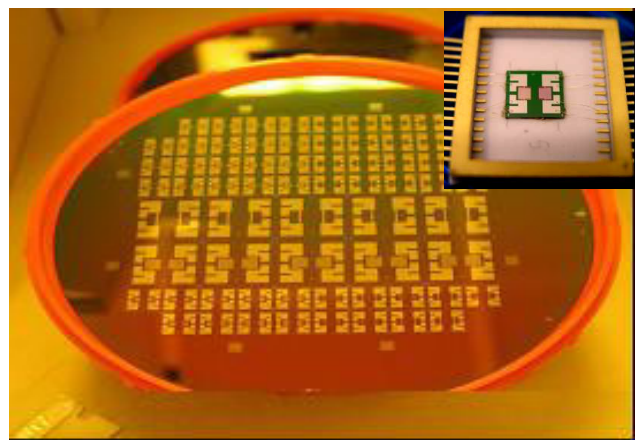

Figure 2. Picture of a completely processed wafer of humidity sensors. The inset photo shows a packaged die for humidity sensor testing.

\section{EXPERIMENTAL RESULTS}

Dies were coated individually with $2 \mu \mathrm{L}$ of $0.01 \mathrm{M}$ Poly Styrene Sulfonic Acid (PSSA) co-polymer. Table 2 shows the water absorption capability of four different formulations of PSSA. Thermal Gravametric Analysis (TGA) was used to determine the weight change of different four different formulations of PSSA. Graphs of the water absorption behavior showed that the $\mathrm{H}+$ co-polymer had a stable water uptake with temperature in comparison with some of the more highly

Table 2. Weight change due to water absorption for various co-polymer PSSA formulations.

\begin{tabular}{|l|l|}
\hline & Percent Weight Change \\
\hline $\mathrm{Li}^{+}$ & $26.9 \%$ \\
\hline $\mathrm{Cs}^{+}$ & $10.5 \%$ \\
\hline $\mathrm{Mg}^{2+}$ & $30.6 \%$ \\
\hline $\mathrm{H}^{+}$ & $18.0 \%$ \\
\hline
\end{tabular}

absorptive formulations. One risk of using a highly adsorptive polymer is the generation of high stresses on the membrane structure causing device failure. The PSSA was applied to the backside the membrane with a small syringe and allowed to dry for 5-10 minutes.

Figure 3 shows a schematic of the test setup used to examine the resonant response of the humidity sensor. The individual dies were placed in a Teflon ${ }^{\mathrm{TM}}$ jig that included a small permanent magnet located underneath the chip, spring-loaded probes, and an interrogation hole for the laser vibrometer. The permanent magnet is approximately $12 \mathrm{~mm}^{3}$ and generates a weak magnetic field of approximately $3 \mathrm{~T}$. This jig was fixed to a port of a stainless steel pressure vessel. The fixture also included a hole which allowed the atmosphere of the chamber to interact with the polymer on the backside of the membrane. The atmosphere of the chamber was controlled through the use of a Dew Point Generation (DPG) system and a chilled mirror humidity sensor. Dry Nitrogen gas was used as the carrier gas for the humidity. A network analyzer in conjunction with a laser vibrometer was used to drive the membrane into resonance and measure the resonant frequency.

Each test consists of two phases: desorption and adsorption. First, a DC power supply was used to desorb the absorbed water in the PSSA using a power of approximately $40 \mathrm{~mW}$. Simultaneously, the Dew Point Generator set to the desired setpoint and its output is checked with the chilled mirror. The chamber humidity is allowed to stabilize during the desorbtion of water from the polymer. Once the sensor has fully desorbed water from the polymer material, the DC power supply is terminated. The polymer is allowed to adsorb humidity from the humidity

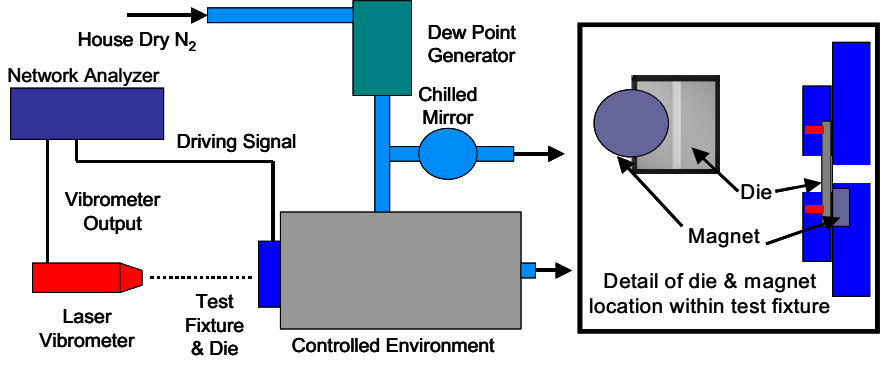

Figure 3. Experimental setup for resonant frequency measurements at specified humidity.

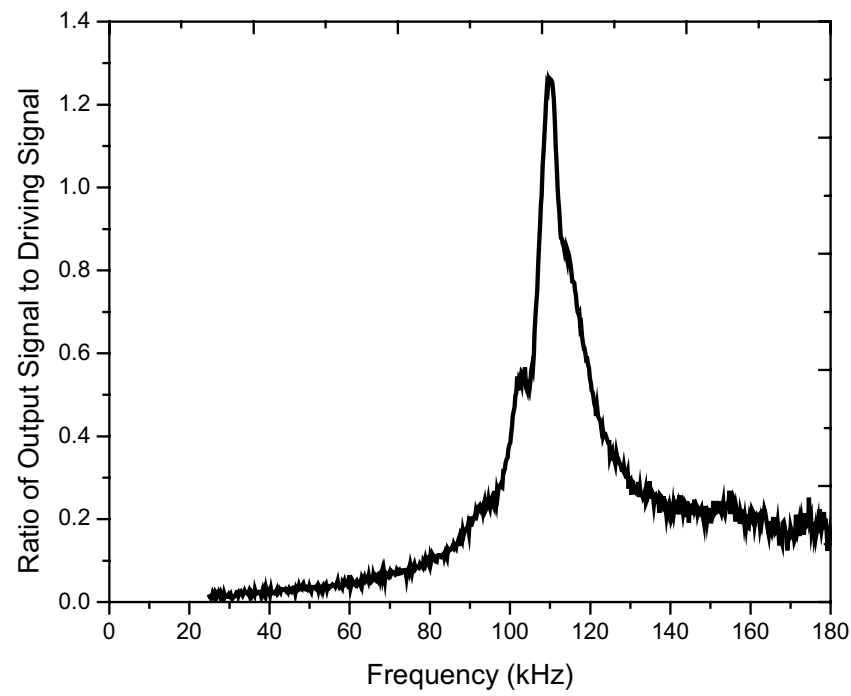

Figure 4. Resonant frequency response for a 1 by $1.3 \mathrm{~mm}$, $0.5 \mu \mathrm{m}$ thick membrane at 217 ppm water. 
Table 3. Humidity Sensor performance with respect to geometry

\begin{tabular}{|c|c|c|c|l|l|}
\hline $\begin{array}{l}\text { Membrane } \\
\text { Area }\left(\mathrm{mm}^{2}\right)\end{array}$ & $\begin{array}{l}\text { Membrane } \\
\text { Thickness } \\
(\mu \mathrm{m})\end{array}$ & $\begin{array}{l}\text { Sensitivity } \\
\left(\mathrm{Hz} /{ }^{\circ} \mathrm{C}\right. \\
\mathrm{DP})\end{array}$ & $\begin{array}{l}\text { Max } \\
\text { Non- } \\
\text { linearity }\end{array}$ & $\begin{array}{l}\text { Max } \\
\text { Hysteresis }\end{array}$ & $\begin{array}{l}\text { Resolution } \\
\left({ }^{\circ} \mathrm{C} \text { DP }\right)\end{array}$ \\
\hline 2.7 & 1.0 & 20.58 & $0.14 \%$ & $0.07 \%$ & 4.37 \\
\hline 2.7 & 0.5 & 69.28 & $0.40 \%$ & $0.40 \%$ & 1.30 \\
\hline 1.3 & 1.0 & 39.85 & $0.37 \%$ & $0.39 \%$ & 2.26 \\
\hline 1.3 & 0.5 & 69.64 & $0.44 \%$ & $0.66 \%$ & 1.29 \\
\hline
\end{tabular}

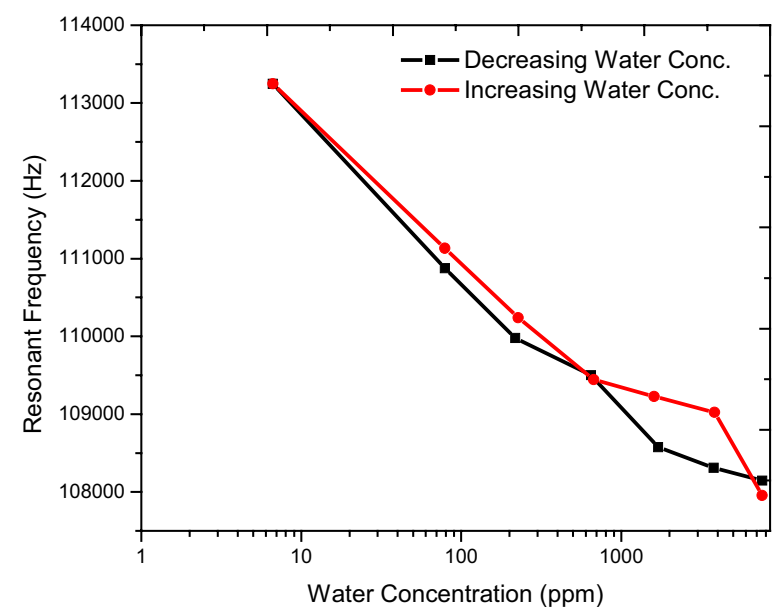

Figure 5. Hysteresis over a 7500 ppm range of a 1 by $1.3 \mathrm{~mm}, 0.5$ um thick membrane structure.

controlled environment for approximately 5 minutes. This time is required to ensure a homogenous environment with our high volume chamber. The network analyzer then drives the membrane into resonance by scanning a range of frequencies between 0 $200 \mathrm{kHz}$ at an output power of $15 \mathrm{dbs}$ @ 1.26 Volts. A measurement is made of the ratio of the output of the vibrometer divided by driving signal generated by the network analyzer.

Figure 4 shows a typical resonant peak for a 1.0 by $1.3 \mathrm{~mm}$ $0.5 \mu \mathrm{m}$ thick membrane. Tests for each design were performed at dew points ranging from $-60^{\circ} \mathrm{C}$ up to $10^{\circ} \mathrm{C}$ at intervals of $10^{\circ} \mathrm{C}$ which corresponds to a range of water concentrations of 7 to $7600 \mathrm{ppm}$. Table 3 summarizes the response for each of the designs in terms of the sensitivity, non-linearity, and hysteresis. Figure 5 summarizes the resonant testing results examining the effect of the membrane geometry on sensitivity. Hysteresis measurements were performed by cycling the humidity from $-60^{\circ} \mathrm{C}$ up to $10^{\circ} \mathrm{C}$ to determine if the membrane or polymer has any memory of its previous state.

Drift of the sensor was tested over a time span of 1 hour and examined the accuracy of the measurements. Measurements were taken at three different humidity levels $\left(-60^{\circ} \mathrm{C},-30^{\circ} \mathrm{C}, 10^{\circ} \mathrm{C}\right)$ to determine if there were any differences in drift at different humidities. Sensor drift was limited to $100 \mathrm{~Hz}$ that did not vary with humidity. This corresponds to a maximum resolution of the 1.0 by $1.3 \mathrm{~mm}, 0.5 \mu \mathrm{m}$ thick membrane of $2.5^{\circ} \mathrm{C}$ dew point.

An analysis of the data suggests that increased sensitivity can be achieved by optimizing the membrane geometry. Reducing the thickness of the membrane improves the sensitivity of the sensor, which is consistent with the conclusions provided by Eq.9. Smaller geometry membranes also improve the sensitivity of the device although this effect becomes minimal at very thin geometries where the effect of thickness is more considerable.

\section{CONCLUSIONS}

Humidity sensors based on resonant frequency shift due to a mass change of polystyrene sulfonic acid were fabricated and tested. Small membrane structures with very thin membranes showed a very linear response over a wide bandwidth and at low dew points. Sensitivities, hysteresis, and resolution of this resonant-based sensor compare very favorably to polyimide sensors based on resonant structures [4] or capacitive schemes [12]. Further testing is necessary determine if sensitivity improvements can be obtained with PSSA formulations that exhibit better water adsorbtive properties such as $\mathrm{Mg}^{2+}$ or $\mathrm{Li}^{+}$. Materials such as self-assembled monolayers or zeolites could also show improved sensitivity and resolution due to their higher surface area and nanomorphology. In order to mass produce humidity sensors based on polyelectrolytes, low cost coating techniques are being developed.

\section{ACKNOWLEDGMENTS}

The authors would like to thank the support and hard work of Joe Krisciunas, Stacey Goodwin, Laura Meyer, Glenn Claydon, and Dennis Cusano.

\section{REFERENCES}

1. T. Hayes and R. Bayrer, "Chemical Sensors: Liquid, Gas, and Biosensors", The Freedonia Group, Inc., April 2002.

2. R. E. Cavicchi, et. al., "Microdifferential Scanning Calorimeter for Combustible Gas Sensing”, Sensors and Actuators B 97 (2004) pp. 22-30.

3. Z. M. Rittersma, "Recent achievements in miniaturized humidity sensors-a review of transduction techniques", Sensors and Actuators A 96 (2002) pp. 196-210.

4. T. Harpster, B. Stark, and K. Najafi, "A passive wireless integrated humidity sensor", Proceedings of the $14^{\text {th }}$ IEEE International Conference on MEMS, Interlaken, Sweden, January 21-25 2001, pp. 553-557.

5. A. Foucaran, et. al., "Porous Silicon Layer Coupled with a Thermoelectric Cooler: A Humidity Sensor", Sensors and Actuators A, 79, (200), pp. 189-193.

6. T. Nitta and S. Hayakawa, "Ceramic Humidity Sensors", IEEE transactions on components, hybrids, and manufacturing technology, Vol. CHMT3, No.2 June 1980.

7. W. H. Tao, et. al., "The humidity sensing properties of Organic Inorganic (AMPS/SiO ${ }_{2}$ ) hybrid materials", Proceedings of IEEE Sensors, Taipei, Taiwan, June 12-14 2002, Vol. 1, pp. 641-646. 8. R. Cunningham and R. Williams, Diffusion in Gases and Porous Media, Plenum Press, New York, 1980.

9. A. Schroth, et. al., "A resonant Polyimide-based humidity sensor", The $8^{\text {th }}$ international conference on solid state sensors and actuators, and Eurosensors IX, Stockholm, Sweden, June 25-29 1995, Vol. 2, pp. 740-742.

10. C. Grimes, D. Kouzoudis, E. Dickey, D. Qian, M. Anderson, R. Shahidain, M. Lindsey, and L. Green, "Magnetoelastic Sensors in Combination with Nanometer-scale Honeycombed Thin Film Ceramic TiO2 for Remote Query Measurement of Humidity", Journal of Applied Physics, 87, 9 (2000).

11. M. Rubinstein, R. H. Colby, A. V. Dobrynin, J.-F. Joanny, "Elastic modulus and equilibrium swelling of Polyelectrolyte Gels", Macromolecules, 29, 398-406, (1996).

12. C. Laville and C. Pellet, "Interdigitated Humidity Sensors for a Portable Clinical Microsystem", IEEE Transactions on Biomedical Engineering, 49, 10, 1162-1167, (2002). 